\title{
TO LANGUAGE LEARNERS
}

IF YOU ARE choosing this book to continue your language studies, you may be familiar with and understand the potential of using a corpus for developing your language ability. As you may know, a corpus (plural: corpora) is a large collection of authentic language which has been collected for a variety of purposes. Researchers use corpora to study language use and language change over time, lexicographers and textbook writers use corpora to develop resources and learning materials, and language learners use corpora to explore language and answer their own questions about language use. You may still be asking, 'But why do these groups choose to use corpora instead of other resources?' There are several answers to this question. First, as stated in its definition, a corpus is a collection of authentic language. These are not examples imagined and created to highlight a particular word or part of speech. These are sentences spoken and written by real language users in real world situations and contexts. If researchers, teachers, and learners are going to study and explore language in order to better understand how it is used, it seems only reasonable that we should look at the actual language people use rather than imaginary examples.

Second, corpora are used to study language because often what people believe about how, when, or why they use a particular word or phrase is often inaccurate or even incorrect. It's simply true that most people rarely consider questions such as 'What is the difference between near-synonyms like hear and listen or beautiful and attractive?' or reflect on the differences between when and while. Thus, it is only natural that people may not give accurate answers to these questions. So, for researchers, instructors, and learners, it can be more useful to search a corpus for data rather trusting our own or another person's intuition about language use.

Third, and related to the prior reason, corpora can help you become an independent and autonomous language learner. Rather than waiting to ask a question to a classmate or instructor, you will be able to ask and answer your own 
questions. Through this process of discovery, your language skills will develop as you carefully read and think about the information you are presented, and you will be able to make informed decisions about language choices.

This text provides a step-by-step, hands-on approach to help you advance your own language ability. As you move through the text, take time to reflect on the findings and complete your own corpus searches.

\section{To the corpus!}

\section{KEY FEATURES}

- Step-by-step illustrated examples to help readers achieve the potential of corpus linguistics for language learning

- Tutorials using a variety of popular corpora

- Opportunities for additional reflection and practice throughout the text

- Tutorials aimed at promoting learner autonomy and self-discovery 